\title{
Shedding Light on Helical Microtubules: Real-Time Observations of Microtubule Self-Assembly by Light Microscopy
}

\author{
Hee-Young Lee, ${ }^{\dagger}$ Hyuntaek Oh, $^{\dagger}$ Jae-Ho Lee, and Srinivasa R. Raghavan* \\ Department of Chemical and Biomolecular Engineering, University of Maryland, College Park, Maryland 20742-2111, United States \\ Supporting Information
}

ABSTRACT: Helical tubules are a fascinating and an intriguing class of self-assemblies. They occur frequently in biology and are believed to be intermediates in formation of gallstones. The pathway by which amphiphiles transform from an initial state of vesicles or micelles into such tubules has puzzled soft matter physicists, and it has raised important questions about the interplay between molecular chirality and self-assembly. Here, for the first time, we demonstrate direct, real-time observations by light microscopy of the pathway to helical microtubules from an initial solution of nanoscale vesicles. The tubules are formed in aqueous mixtures of the

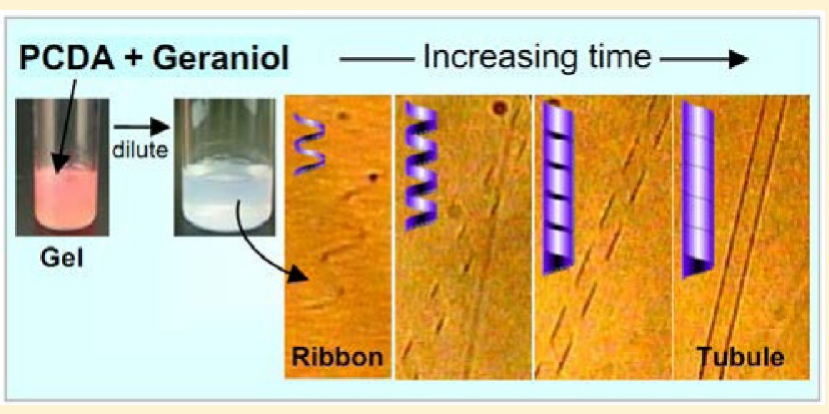
single-tailed diacetylenic surfactant, 10,12-pentacosadiynoic acid (PCDA), and a short-chain alcohol. The stepwise process involves nucleation of thin helical microribbons from the vesicle solution. These ribbons then thicken, rearrange, and fold into closed tubules. Subsequently, most tubules further rearrange into plate-like structures, and once again, we are able to visualize this process in real time. A notable aspect of the above system is that the precursors are achiral; yet, the tubules are formed from helical ribbons. Our study provides new insights into tubule formation that will be valuable in clarifying and refining theoretical models for these fascinating structures.

\section{INTRODUCTION}

The self-assembly of amphiphiles in dilute aqueous solution is a well-known phenomenon, and it usually leads to assemblies such as micelles and vesicles. ${ }^{1,2}$ A far more unusual type of structure can also be assembled from amphiphiles, and these are hollow cylinders or tubules with diameters in the nano- to microscale. In the simplest case, tubules can be envisioned to form by taking an amphiphilic bilayer sheet and twisting and wrapping this sheet into a helical tube. ${ }^{3}$ The first reports of such tubules date back to the $1980 \mathrm{~s}^{4}$ however, tubules continue to remain rather mysterious structures because they are rarely seen in conventional surfactant or lipid solutions. In turn, their morphology and assembly mechanisms are much less understood.

The study of tubules essentially began with a paper by Yager and Schoen reporting such structures in a solution of a diacetylenic phospholipid. ${ }^{4}$ Since that time, tubules have been reported in solutions containing phospholipids or glycolipids, ${ }^{5-10}$ gemini surfactants, ${ }^{11}$ and peptide amphiphiles. ${ }^{12-15}$ The field of tubules has assumed importance in soft matter physics owing to the connection between molecular chirality and self-assembly. ${ }^{5,16}$ Tubules may also have biological relevance: for example, they have been found in solutions of human bile ${ }^{17-19}$ and are believed to be intermediate structures in the formation of gallstones. ${ }^{17,18}$ In addition to the scientific implications, tubules have also received extensive consideration for applications such as controlled release and drug delivery and as templates for materials synthesis. ${ }^{5,20,21}$
The mechanism and pathway for tubule formation has been addressed in both experimental ${ }^{15,22-24}$ and theoretical ${ }^{16,25-29}$ studies. Researchers have focused on elucidating how initial structures such as vesicles or micelles transform into helical ribbons and finally into helical tubules. Because tubules and their precursors typically have nanoscale dimensions at the outset, direct observation of tubule formation requires the use of transmission or scanning electron microscopy (TEM or SEM) or atomic force microscopy (AFM). With these techniques it is difficult or impossible to track individual tubules in real time; one only obtains snapshots of the entire system at different time points during tubule formation. Optical (light) microscopy, either in bright-field or in fluorsecence mode, is ideally suited to real-time visualization of assembly processes. However, to our knowledge, current systems do not allow in situ tracking of individual tubules by light microscopy, partly because the sizes involved are typically too small (below the optical resolution). Moreover, for real-time tracking to be truly informative, the system should evolve slowly so that the various steps or morphological transitions can be clearly distinguished. At the same time, if the evolution is too slow, the structures cannot be tracked reliably because they will tend to drift away from the field of view due to Brownian motion or convection.

Received: April 2, 2012

Published: August 21, 2012 
In this paper, we report a new class of amphiphile-based tubules of microsized diameters and lengths, which can be directly observed by light microscopy. Thereby, we have been able to track in real time the pathway from an initial state of vesicles into helical ribbons and then into hollow tubules. The conditions (temperature, concentration) have been modulated in such a way that the whole process occurs over several hours, which is sufficiently long to enable clear identification of intermediate states along the way. Additionally, we find that most tubules eventually transform into plate-like structures over approximately 2 days. All morphological transitions have been tracked in real time for individual structures, i.e., we can see the same entity transform over time. To our knowledge, such realtime observations of tubule formation and rearrangement into plates have not been reported in the literature. Together, our studies provide a variety of new insights into the formation mechanism and properties of helical microtubules.

\section{RESULTS AND DISCUSSION}

The tubules studied here are formed in mixtures of a singletailed diacetylenic surfactant, 10,12-pentacosadiynoic acid (PCDA), and an alcohol such as geraniol (Figure 1). Both

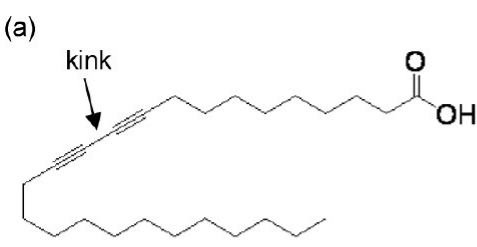

10,12-pentacosadiynoic acid (PCDA)

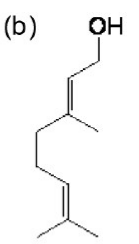

geraniol

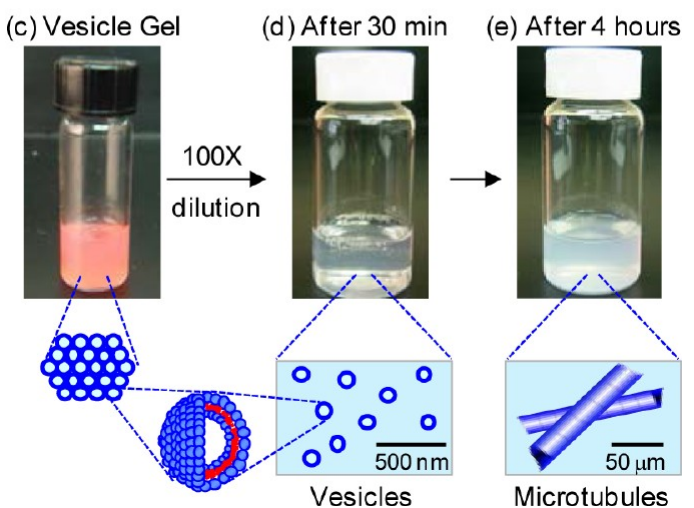

Figure 1. Molecular structures of (a) 10,12-pentacosadiynoic acid (PCDA) and (b) geraniol. (c) Photograph of the gel formed by mixing 11 wt \% PCDA and 11 wt \% geraniol. Gel is expected to consist of densely packed vesicles. (d) Upon $100 \times$ dilution of the gel with water, a bluish solution is initially obtained, consisting of dilute vesicles. (e) With time, the sample becomes increasingly turbid and hollow tubules with microsized diameters and lengths appear in it.

PCDA and geraniol are commercially available and relatively inexpensive compounds, and thus, our tubule studies can be replicated easily in any laboratory. Note that the diacetylenic phospholipid 1,2-bis(10,12-tricosadiynoyl)-sn-glycero-3-phosphocholine $\left(\mathrm{DC}_{8,9} \mathrm{PC}\right)$ was one of the first amphiphiles shown to form tubules, ${ }^{5}$ and this molecule had a chiral center in the lipid headgroup and a kink in each of its two tails due to the triple bonds. Tubules of $\mathrm{DC}_{8,9} \mathrm{PC}$ were formed by dissolving the lipid above its melting temperature in alcohol/ water mixtures and then cooling this solution down to room temperature, whereupon tubules of $500 \mathrm{~nm}$ diameter appeared. PCDA is a single-tailed analog of $\mathrm{DC}_{8,9} \mathrm{PC}$ with a similar tail structure; however, its headgroup does not have a chiral center. To form tubules, PCDA has to be combined with a cosurfactant such as geraniol, an alcohol found in nature as the primary component of rose oil. (Other medium-chain alcohols like octanol or decanol can also be used instead of geraniol.) Our method to form PCDA-geraniol tubules does not involve cooling a hot solution. Instead, a concentrated mixture of PCDA and geraniol (11 wt \% each) is initially created in deionized water. This sample is a translucent pink gel, as shown in Figure 1c. The gel holds its weight in an inverted vial, and the gel-like character is confirmed by dynamic rheology (Supporting Information, Figure S1). Such gels are stable for more than 1 year when stored in the dark. On the basis of studies by others with similar surfactant/alcohol mixtures, we can surmise that the gel behavior is due to an array of densely packed unilamellar vesicles. ${ }^{30-33}$

Tubules are formed when the above PCDA/geraniol gel is diluted with water. We should note that if we simply combined low concentrations of PCDA and geraniol corresponding to a dilute sample, the mixture would remain a multiphase dispersion, i.e., the molecules would not dissolve. Indeed, both PCDA and geraniol are insoluble in water by themselves; however, the mixed gel is homogeneous. Let us now examine the effect of diluting the PCDA/geraniol gel shown in Figure 1c $(0.1 \mathrm{~g})$ with an excess of water $(10 \mathrm{~g}$, i.e., $100 \times$ dilution $)$. The sample initially becomes a clear, bluish solution as shown in Figure $1 \mathrm{~d}$. The bluish color is an indication of vesicles, and dynamic light scattering (DLS) reveals that the vesicles have a diameter of about $100 \mathrm{~nm}$. TEM additionally confirms the presence of spherical vesicles in this sample (Supporting Information, Figure S2), and the sizes from TEM are consistent with that from DLS. As time progresses, the bluish vesicle sample becomes more turbid (Figure 1e), indicating that larger structures are formed, and these larger structures are shown below to be tubules.

We observed the sample under a light microscope at a temperature of $40{ }^{\circ} \mathrm{C}$ and as a function of time after dilution. The higher temperature of $40{ }^{\circ} \mathrm{C}$ (as compared to room temperature) was chosen because it slowed down the structural evolution and thereby allowed careful examination of the structures. Representative micrographs at different time points are shown in Figure 2. At short times (0-1 h after dilution), the structures (i.e., spherical vesicles) are too small to be distinctly observed by light microscopy. Around the $1 \mathrm{~h}$ mark, very thin helical ribbons with microsized diameters and lengths emerge (Figure 2a). Around the $2 \mathrm{~h}$ mark, the helical ribbons widen (Figure $2 \mathrm{~b}$ ), and by the $4 \mathrm{~h}$ mark, these rearrange into hollow tubules with diameters of 3-22 $\mu \mathrm{m}$ and lengths exceeding 90 $\mu \mathrm{m}$ (Figure $2 \mathrm{c}$ ). After 6-7 h, many of the tubules begin to rearrange and transform slowly (over the next $40 \mathrm{~h}$ ) into rigid plate or sheet-like structures. The latter are the dominant structure in the sample after 2 days (Figure 2d); however, a few tubules are still found in this sample. Our images show that ribbons and tubules or tubules and plates often coexist, indicating that the times of formation and disruption of each structure are quite different.

The helical ribbons were characterized by their pitch angle $(\psi)$ and diameter $(d)$. For this we selected 500 helical ribbons from images between 1 and $4 \mathrm{~h}$ after dilution of the gel and measured the pitch and diameter from the images, as shown in 

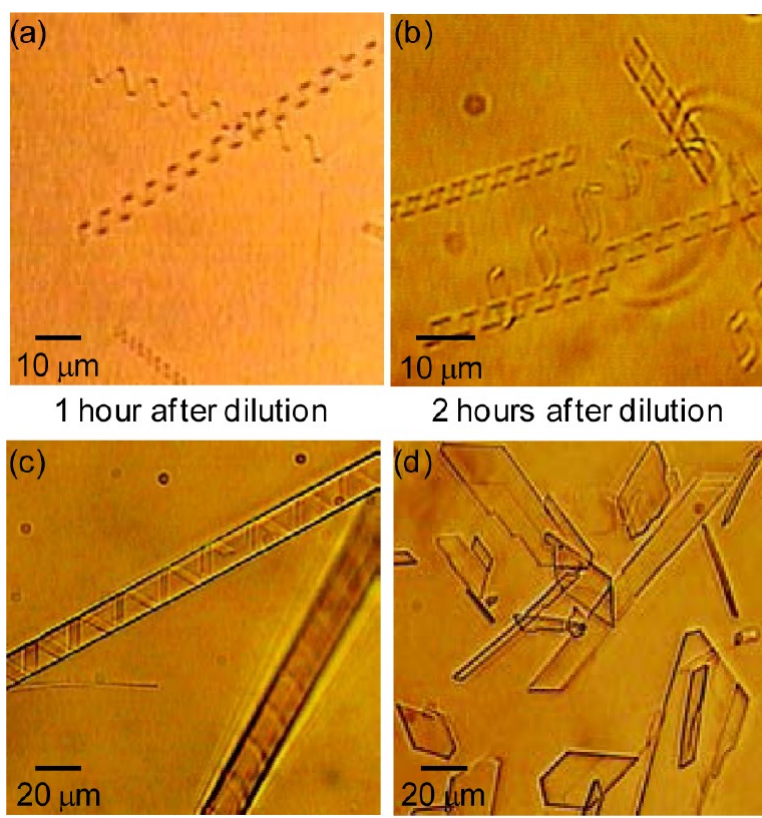

4 hours after dilution

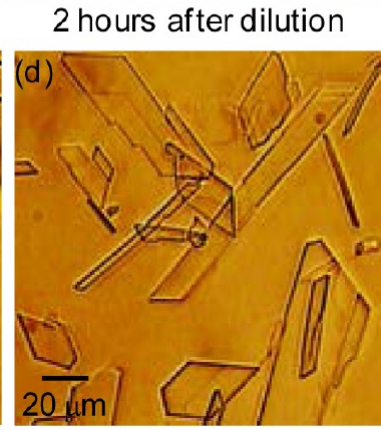

24 hours after dilution

Figure 2. Optical micrographs showing the evolution of structures in a PCDA/geraniol sample as a function of time after dilution of the gel. (a) At the $1 \mathrm{~h}$ mark, thin helical ribbons are seen. (b) These ribbons become wider and (c) rearrange into hollow tubules with microsized diameters and lengths. (d) Within $24 \mathrm{~h}$, most tubules turn into rigid plate or sheet-like structures.

Figure 3. The results (top plot of Figure 3) show that most of the ribbons, regardless of their diameter, have pitch angles of
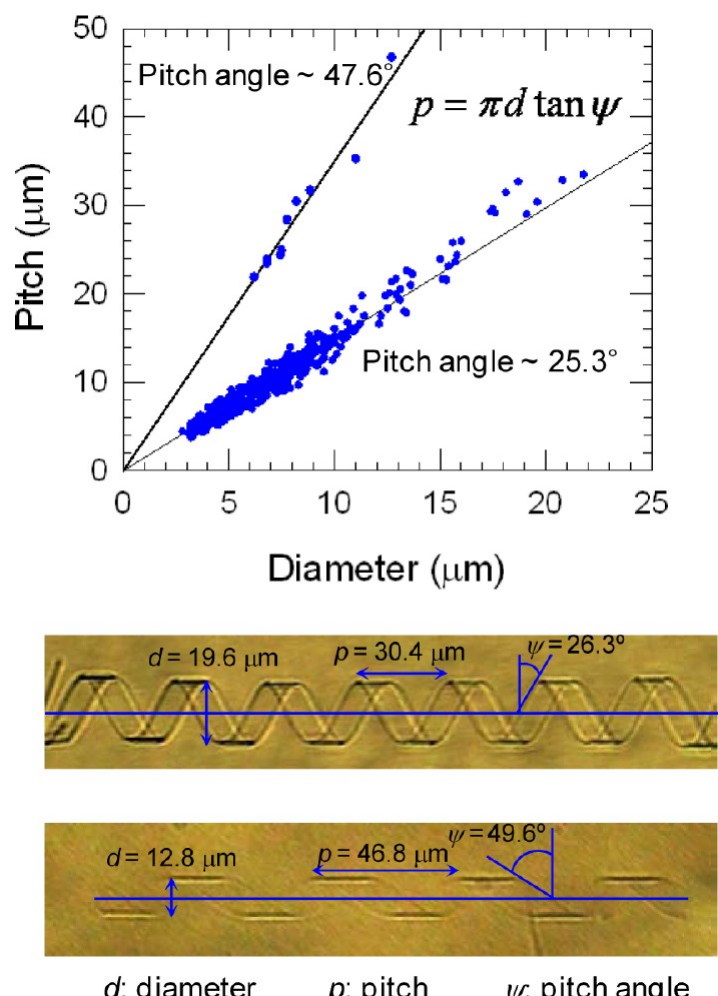

Figure 3. Pitch vs diameter for helical ribbons (top plot). Pitch angles were found to be $25.3 \pm 5.0^{\circ}$ for most tubules (example shown in top image) and $47.6 \pm 2.2^{\circ}$ for a few others (example in bottom image).
$25.3 \pm 5.0^{\circ}$ while a few ribbons have pitch angles of $47.6 \pm$ $2.2^{\circ}$. Pitch angles around $25^{\circ}$ have been seen before for tubules formed by diacetylenic phospholipids. ${ }^{8,34} \mathrm{We}$ also examined 500 helical ribbons in an attempt to distinguish whether they were right- or left-handed (see Supporting Information, Figure S3). The ratio of right-handed ribbons (245) to left-handed ribbons (255) was 0.96. It is well known that molecular chirality is a key to having favored handedness of helical ribbons. ${ }^{35,36}$ Since both PCDA and geraniol are achiral, our finding of near-equal handedness is quite reasonable. Note, however, that molecular chirality is not essential for tubule formation; instead, the kink in PCDA tails is the crucial factor that dictates tubule formation, as noted previously for achiral diacetylenic lipids. ${ }^{9}$

Next, we address the pathway from helical ribbons to tubules. To follow this pathway, we directly observed and tracked individual structures over time. Figure 4 presents a series of optical micrographs showing a transition of an individual helical ribbon to a tubule. This helical ribbon was initially seen after 60 min of dilution of the PCDA/geraniol gel, and at this point it has a diameter of $7.9 \mu \mathrm{m}$ and a pitch of $24.9 \mu \mathrm{m}$ (Figure $4 \mathrm{a}$ ). Between 100 (Figure 4b) and $140 \mathrm{~min}$ (Figure 4c) the length of the ribbon increases while its diameter, pitch, and width remain constant. Between 180 (Figure 4d) and $210 \mathrm{~min}$ (Figure $4 \mathrm{e}$ ) the ribbon grows laterally, i.e., in its width, while also continuing to grow in length. Between 240 (Figure 4f) and $270 \mathrm{~min}$ (Figure 4g) the width continues to increase, and in the process, adjacent portions of the ribbon merge by removing the gaps between the turns. This ultimately results in a closed hollow tubule (Figure $4 \mathrm{~g}$ ), and the entire process is schematically shown in Figure 4h. SEM images (Figure 4i) confirm the hollow structure of the tubule, and the tubule surface is found to be very smooth, indicating that the surface is created by smooth merging of ribbon portions.

A crucial point from Figure 4 is that the diameter and pitch of the final tubule are identical to those of the initial helical ribbon. This finding allows us to discriminate between existing models for tubule formation. In the closing-pitch model, ${ }^{17,37}$ the pitch of the helical ribbon gradually shortens until a tubule is formed while the width of the ribbon remains unchanged. In the growing-width model, ${ }^{22}$ on the other hand, the width of the ribbon grows until a tubule is created while the pitch and diameter of the ribbon remain unaltered. Our observations for PCDA/geraniol tubules are undoubtedly consistent with the growing-width model and not the closing-pitch model. This is the case regardless of tubule diameter or pitch angle. Figure S4 (Supporting Information) shows a similar set of images as in Figure 4 for a helical ribbon with a larger diameter $(17.5 \mu \mathrm{m})$ that closes into a tubule. Overall, we believe these are some of the clearest real-time observations of an individual ribbon evolving into a tubule.

An interesting wrinkle in the formation process was noted for some tubules (Figure 5). In Figure 5a, we see a helical ribbon that is about to close into a tubule: note that adjacent portions of the ribbon are very close to each other. However, when the tubule is formed (Figure $5 \mathrm{~b}$ ), the ribbon portions do not smoothly merge but instead undergo partial overlap. The overlapping areas are indicated by arrows in Figure $5 \mathrm{~b}$ and show up as helical bands. This is also seen in the SEM image in Figure 5c. Thus, the thickness of the tubule wall in the overlapping areas is higher than in the nonoverlapping areas. Interestingly, tubules with overlapped ribbon portions seem to retain their stability in solution for a longer time (1-2 days) 

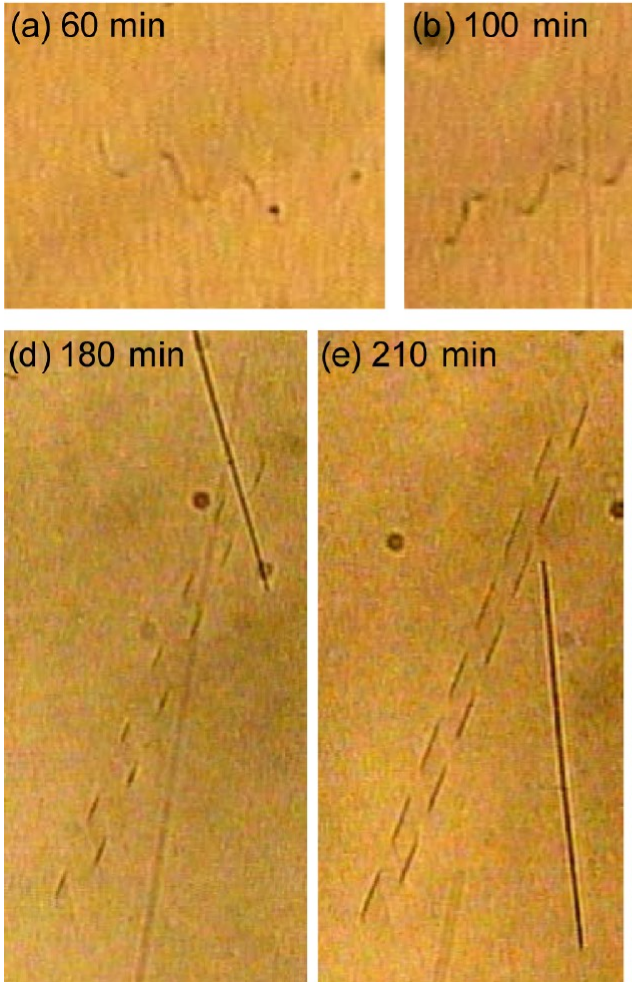
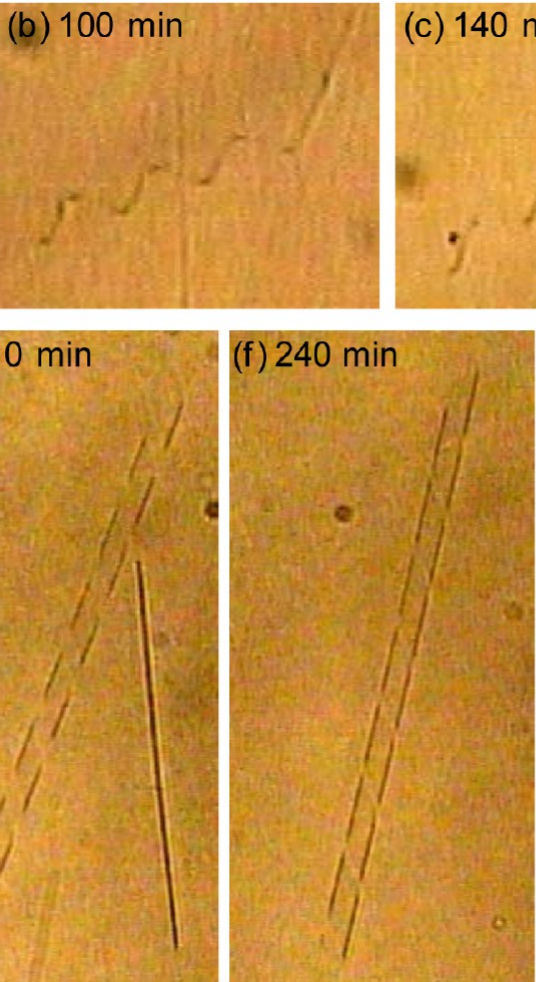

$240 \mathrm{~min}$

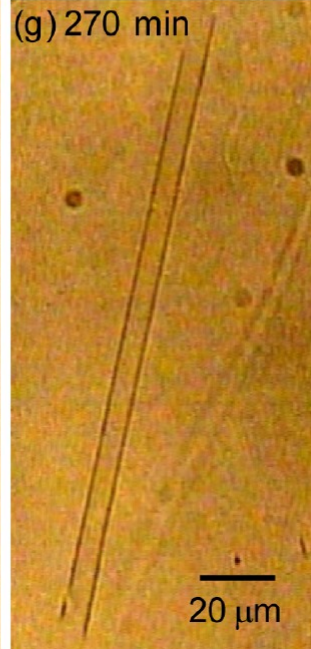

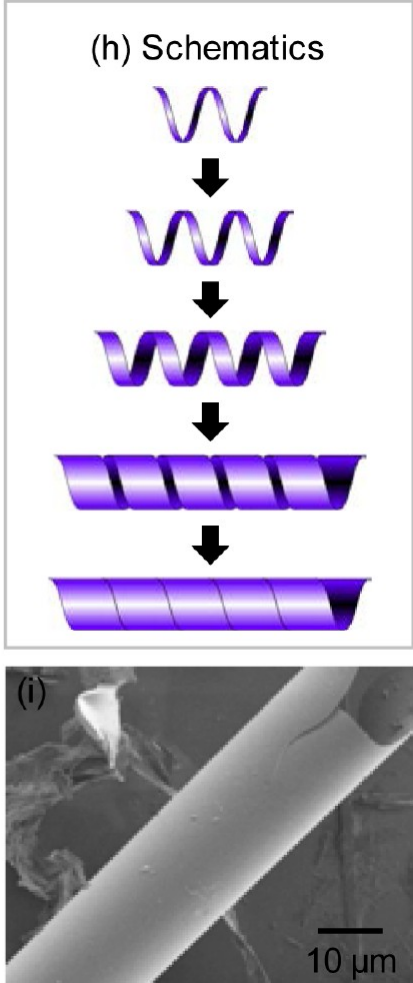

Figure 4. Optical micrographs in real time tracing the transformation of a helical ribbon to a closed hollow tubule: (a) 60 min after dilution of the PCDA/geraniol gel, a thin helical ribbon is identified. This helical ribbon grows longer, as shown by the images at 100 (b) and 140 min (c), while its diameter, width, and pitch remain unchanged. Around $180 \mathrm{~min}(\mathrm{~d})$, the ribbon width begins to increase, and this is further seen at 210 (e) and 240 $\min (\mathrm{f})$. As the width increases, the gaps between the turns are eliminated, resulting in a closed hollow tubule around $270 \mathrm{~min}(\mathrm{~g})$. Schematics of tubule formation by this "growing width" mechanism are presented in Figure 4h: note that the diameter and helical pitch remain constant during the entire process. SEM image in Figure $4 \mathrm{i}$ shows a hollow microtubule with a smooth surface.
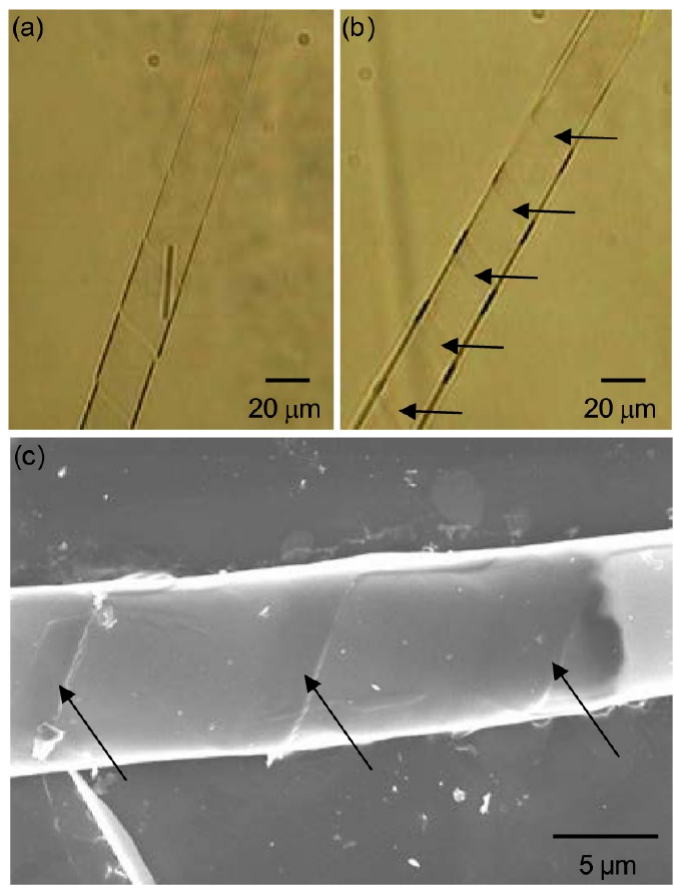

Figure 5. Tubule formed by overlapping of helical ribbon portions. (a) Optical image shows a helical ribbon whose segments have almost merged to form a closed tubule. However, rather than merging smoothly, the segments partially overlap as they merge to form the tubule (b) (arrows indicate the overlapping parts). SEM image of such a tubule $(c)$, with the overlap points again indicated by arrows. than those with smooth walls that were formed by merging of ribbons. We speculate that multiple bilayers in the overlapping areas may contribute to additional stability of the tubules (e.g., the resulting tubules could be more difficult to unravel or unwind; see Figure 6).

Finally, we address the process by which tubules are converted into plate-like structures. ${ }^{17,18}$ As mentioned earlier, once tubules are formed, they are stable in solution for time scales ranging from several hours to 2 days. However, most tubules are eventually converted into plates. We directly observed individual tubules in real time by light microscopy and tracked their transition into plates. Figure 6 presents a series of optical micrographs showing such a transition. The starting point is an intact hollow tubule (Figure 6a) with a diameter of $4.3 \mu \mathrm{m}$ and a length of $148 \mu \mathrm{m}$. Within $10 \mathrm{~min}$, a fracture or kink appears in the middle of the tubule at the point shown by the arrow (Figure $6 \mathrm{~b}$ ). The tubule then begins to unravel at this point and opens up into a helical ribbon with a pitch angle of $\sim 23^{\circ}$ and eight turns (Figure $6 \mathrm{c}$ ). Note that two short segments of intact tubule flank the ribbon portion. Thereafter, the middle ribbon portion expands in diameter and in width, and in doing so the number of turns in the ribbon decrease from eight to five (Figure 6d). By the 285 min mark (Figure $6 \mathrm{e})$, the tubule has almost completely unraveled and most of it is now a large sheet-like ribbon with a diameter and width exceeding $20 \mu \mathrm{m}$ and only taking up one and a half turns. The sheet is rather stiff: note that it does not bend easily, and it is more like a hard solid than a flexible ribbon. This is also shown by the SEM image in Figure of and by the additional optical images in the Supporting Information, Figure S5. Eventually, 

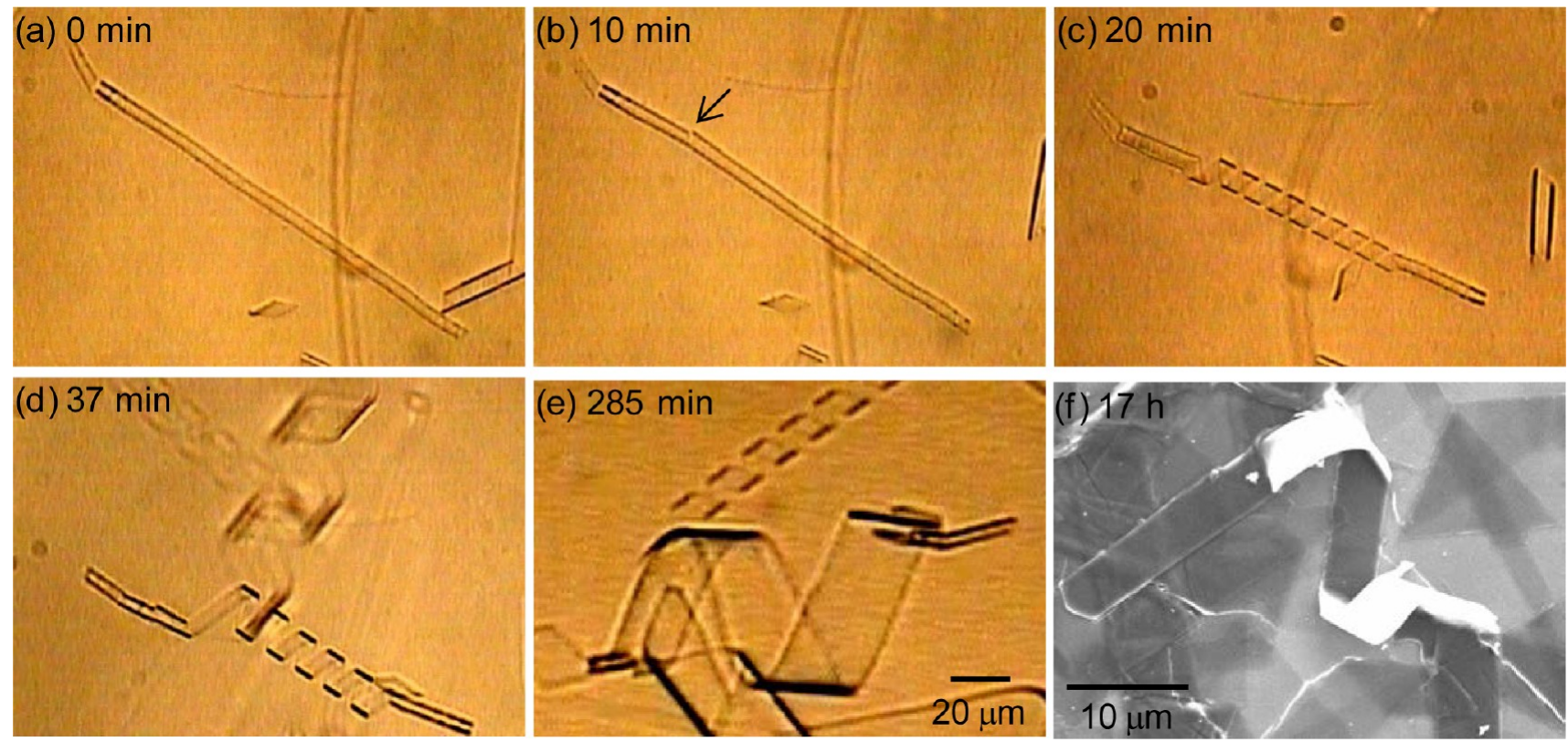

Figure 6. Optical micrographs in real time tracing conversion of tubules to plate or sheet-like structures. (a) Closed tubule is identified for observation. (b) Within $10 \mathrm{~min}$, a fracture is observed to be initiated in the tubule at the point indicated by the arrow. Tubule begins to unravel at this point, resulting in a helical ribbon portion in the middle (c). This ribbon portion grows in width while the number of turns decrease, as shown by $\mathrm{d}$ and e. By $285 \mathrm{~min}$ (e), the original tubule has been converted into a stiff sheet-like structure. (f) SEM image confirms the stiff nature of this structure, which is brittle and eventually breaks into discrete objects.

these stiff sheets, being brittle, break up into the discrete irregular objects (plates) shown in Figure 2d. Because the breaking process is much slower, it has proved difficult to capture images of a plate as it is breaking; however, we do see sheets with tears or cracks in them (Supporting Information, Figure S5b), and presumably these cracks propagate until the structure fractures.

A similar sequence of events was seen with other tubules as they converted to sheets or plates. The unraveling of a tubule back to its constituent helical ribbon structure was the starting point for most tubule-to-plate conversions events we observed. However, the unraveling does not always occur in the middle of a tubule; it can also occur in some cases at a tubule end, as shown in the Supporting Information, Figure S6. In general, unraveling is expected to occur at "defect sites" along the tubule. When a tubule forms by merging of helical ribbon segments, any point at which such merging is imperfect could be a defect site and may be the point at which the kink in Figure $6 \mathrm{~b}$ is initiated, leading to unraveling of the tubule. It is interesting to speculate why the unraveled ribbon or sheet in Figure $6 \mathrm{e}$ is so much more stiff and brittle than the original tubule. Possibly, the alkyl tails in the stiff sheet may be in a frozen state and hence ordered with respect to each othermuch more so than in the initial tubule. In other words, the molecules in the sheet would be arranged much like in a macroscopic crystal.

To our knowledge, our study is the first to identify the steps in the tubule-to-plate conversion process. The finding that this process starts with the unraveling of tubules at a fracture point is significant, and it shows the value of direct, real-time microscopic investigations.

\section{CONCLUSIONS}

In this paper, we studied tubules formed by combining two achiral molecules: a single-tailed diacetylenic surfactant
(PCDA) and an alcohol, viz. geraniol. The tubules were formed isothermally by diluting a gel of PCDA/geraniol. The physical chemistry of these tubules (e.g., effect of amphiphile concentration, alcohol type, temperature) is itself interesting, as is the fact that the tubules are made from achiral precursors. However, those aspects are not the focus here; rather the focus is on the fact that tubule formation under these conditions can be followed in real time by light microscopy. Through these real-time studies we discovered a number of fascinating facts about the tubules. The initial precursors are helical ribbons, and these close into tubules via the "growing width" mechanism. Most tubules undergo smooth merging of adjacent helical turns, but in some cases, the turns overlap partially. We also discovered how the tubules convert into plates-this involves the unraveling of closed tubules at a fracture point, followed by widening and stiffening of the resulting ribbon. Our findings also raise many new questions. For example, how can we stabilize the tubule state and ensure that it does not unravel into plates? These aspects may have relevance for understanding and preventing the formation of gallstones.

\section{MATERIALS AND METHODS}

Materials. 10,12-Pentacosadiynoic acid (PCDA) was purchased from GFS Chemicals. PCDA was purified by dissolving in chloroform and filtering out the insoluble polymerized PCDA. Chloroform was then removed by drying the sample under a fume hood for $24 \mathrm{~h}$ and then drying in a vacuum oven for at least $48 \mathrm{~h}$. The obtained PCDA powder was ground with an agate mortar and kept in the refrigerator before use. Geraniol (98\% purity) was obtained from Sigma-Aldrich and sodium hydroxide $(\mathrm{NaOH})$ pellets ( $99 \%$ purity) was obtained from J. T. Baker. Ultrapure deionized water from a Millipore water purification system was used in preparing samples.

Sample Preparation. PCDA-geraniol tubules were prepared as follows. First, a slight excess of a molar equivalent of 5 wt $\% \mathrm{NaOH}$ solution was added to $11 \mathrm{wt} \%$ PCDA in deionized water, and then 11 wt $\%$ geraniol was added. After vigorous mixing with a vortex mixer, the sample was wrapped with aluminum foil to prevent PCDA 
polymerization by light. The sample was kept at room temperature, whereupon it became a homogeneous pink gel. When this gel $(0.1 \mathrm{~g})$ was diluted with excess water $(10 \mathrm{~g})$, tubules were formed in the sample. The $\mathrm{pH}$ of this sample was around 9. The diluted sample was wrapped with aluminum foil and placed in a $40{ }^{\circ} \mathrm{C}$ water bath until use.

Optical (Light) Microscopy. A Zeiss Axiovert 135TV inverted light microscope equipped with the Motic ImagePlus imaging system was used for high-quality transmission microscopy. To investigate the in situ formation of tubules, the diluted sample from above was loaded in a rectangular glass capillary with an inner thickness of $0.3 \mathrm{~mm}$, and this was mounted onto the microscope. A hot stage linked to a Physitemp TS-4 ER temperature controller was used to control the temperature of the capillary while imaging. The temperature of the hot stage was maintained at $40{ }^{\circ} \mathrm{C}$.

Rheological Studies. Steady and dynamic rheological experiments were performed on a Rheometrics RDA III strain-controlled rheometer. A cone-and-plate geometry of $25 \mathrm{~mm}$ diameter and 0.04 rad cone angle was used. Dynamic frequency spectra were obtained in the linear viscoelastic regime of the samples, as determined by dynamic strain sweep experiments.

Transmission Electron Microscope (TEM). TEM was conducted on a Jeol JEM 2100 microscope at $80 \mathrm{KeV}$ with negative staining using uranyl acetate. Two hundred mesh Formvar-coated copper grids were dipped into the sample and then dried for about $2 \mathrm{~h}$. One percent uranyl acetate was added to the sample and dried again overnight in a fume hood before imaging.

Scanning Electron Microscope (SEM). The surface of a tubule was investigated using SEM (Hitachi SU-70) at $5 \mathrm{kV}$. Sample was dropped on a glass slide and freeze dried. The sample-deposited glass slide was coated by carbon with a Balzer MED010 carbon coater before SEM imaging.

Dynamic Light Scattering (DLS). To measure the sizes of vesicles, a Photocor-FC light scattering instrument was used at a scattering angle of $90^{\circ}$. The instrument was equipped with a $5 \mathrm{~mW}$ laser source at $633 \mathrm{~nm}$ and a logarithmic correlator. From the autocorrelation function, an average diffusion coefficient was extracted, and from this, the hydrodynamic size was calculated through the Stokes-Einstein relationship.

\section{ASSOCIATED CONTENT}

\section{S Supporting Information}

Data from rheology, TEM, and additional images from light microscopy. This material is available free of charge via the Internet at http://pubs.acs.org.

\section{AUTHOR INFORMATION}

\section{Corresponding Author}

E-mail: sraghava@umd.edu

\section{Author Contributions}

${ }^{\dagger}$ These authors contributed equally.

\section{Notes}

The authors declare no competing financial interest.

\section{ACKNOWLEDGMENTS}

This work was partially funded by a grant from NSF-Chemistry. We acknowledge the Maryland NanoCenter for facilitating the SEM and TEM work. We also acknowledge helpful discussions on this work with Dr. Dganit Danino from Technion-IIT.

\section{REFERENCES}

(1) Israelachvili, J. N. Intermolecular and Surface Forces, 2nd ed.; Academic Press: London, 1991.

(2) Evans, D. F.; Wennerstrom, H. The Colloidal Domain: Where Physics, Chemistry, Biology, and Technology Meet; Wiley-VCH: New York, 2001.
(3) Shimizu, T.; Masuda, M.; Minamikawa, H. Chem. Rev. 2005, 105, 1401-1443.

(4) Yager, P.; Schoen, P. E. Mol. Cryst. Liq. Cryst. 1984, 106, 371381.

(5) Schnur, J. M. Science 1993, 262, 1669-1676.

(6) Frankel, D. A.; Obrien, D. F. J. Am. Chem. Soc. 1994, 116, 10057-10069.

(7) Spector, M. S.; Selinger, J. V.; Singh, A.; Rodriguez, J. M.; Price, R. R.; Schnur, J. M. Langmuir 1998, 14, 3493-3500.

(8) Lvov, Y. M.; Price, R. R.; Selinger, J. V.; Singh, A.; Spector, M. S.; Schnur, J. M. Langmuir 2000, 16, 5932-5935.

(9) Pakhomov, S.; Hammer, R. P.; Mishra, B. K.; Thomas, B. N. Proc. Natl. Acad. Sci. U.S.A. 2003, 100, 3040-3042.

(10) John, G.; Masuda, M.; Okada, Y.; Yase, K.; Shimizu, T. Adv. Mater. 2001, 13, 715-718.

(11) Oda, R.; Huc, I.; Schmutz, M.; Candau, S. J.; MacKintosh, F. C. Nature 1999, 399, 566-569.

(12) Vauthey, S.; Santoso, S.; Gong, H. Y.; Watson, N.; Zhang, S. G. Proc. Natl. Acad. Sci. U.S.A. 2002, 99, 5355-5360.

(13) Lu, K.; Jacob, J.; Thiyagarajan, P.; Conticello, V. P.; Lynn, D. G. J. Am. Chem. Soc. 2003, 125, 6391-6393.

(14) Reches, M.; Gazit, E. Science 2003, 300, 625-627.

(15) Ziserman, L.; Lee, H. Y.; Raghavan, S. R.; Mor, A.; Danino, D. J. Am. Chem. Soc. 2011, 133, 2511-2517.

(16) Selinger, J. V.; Spector, M. S.; Schnur, J. M. J. Phys. Chem. B. 2001, 105, 7157-7169.

(17) Chung, D. S.; Benedek, G. B.; Konikoff, F. M.; Donovan, J. M. Proc. Natl. Acad. Sci. U.S.A. 1993, 90, 11341-11345.

(18) Zastavker, Y. V.; Asherie, N.; Lomakin, A.; Pande, J.; Donovan, J. M.; Schnur, J. M.; Benedek, G. B. Proc. Natl. Acad. Sci. U.S.A. 1999, 96, 7883-7887.

(19) Portincasa, P.; Moschetta, A.; Palasciano, G. Lancet 2006, 368, 230-239.

(20) Markowitz, M.; Baral, S.; Brandow, S.; Singh, A. Thin Solid Films 1993, 224, 242-247.

(21) Zhou, Y.; Kogiso, M.; Asakawa, M.; Dong, S.; Kiyama, R.; Shimizu, T. Adv. Mater. 2009, 21, 1742-1745.

(22) Nakashima, N.; Asakuma, S.; Kunitake, T. J. Am. Chem. Soc. 1985, 107, 509-510.

(23) Thomas, B. N.; Lindemann, C. M.; Clark, N. A. Phys. Rev. E 1999, 59, 3040-3047.

(24) Adamcik, J.; Castelletto, V.; Bolisetty, S.; Hamley, I. W.; Mezzenga, R. Angew. Chem., Int. Ed. 2011, 50, 5495-5498.

(25) Selinger, J. V.; MacKintosh, F. C.; Schnur, J. M. Phys. Rev. E 1996, 53, 3804-3818.

(26) Seifert, U.; Shillcock, J.; Nelson, P. Phys. Rev. Lett. 1996, 77, 5237-5240.

(27) Komura, S.; Zhong-can, O. Y. Phys. Rev. Lett. 1998, 81, 473476.

(28) Aime, C.; Tamoto, R.; Satoh, T.; Grelard, A.; Dufourc, E. J.; Buffeteau, T.; Ihara, H.; Oda, R. Langmuir 2009, 25, 8489-8496.

(29) Ziserman, L.; Mor, A.; Harries, D.; Danino, D. Phys. Rev. Lett. 2011, 106, 238105.

(30) Gradzielski, M.; Muller, M.; Bergmeier, M.; Hoffmann, H.; Hoinkis, E. J. Phys. Chem. B. 1999, 103, 1416-1424.

(31) Hoffmann, H.; Thunig, C.; Schmiedel, P.; Munkert, U. Langmuir 1994, 10, 3972-3981.

(32) Panizza, P.; Roux, D.; Vuillaume, V.; Lu, C. Y. D.; Cates, M. E. Langmuir 1996, 12, 248-252.

(33) de Haas, K. H.; Blom, C.; vandenEnde, D.; Duits, M. H. G.; Haveman, B.; Mellema, J. Langmuir 1997, 13, 6658-6668.

(34) Mahajan, N.; Zhao, Y.; Du, T. B.; Fang, J. Y. Langmuir 2006, 22, 1973-1975.

(35) Schnur, J. M.; Ratna, B. R.; Selinger, J. V.; Singh, A.; Jyothi, G.; Easwaran, K. R. K. Science 1994, 264, 945-947.

(36) Spector, M. S.; Easwaran, K. R. K.; Jyothi, G.; Selinger, J. V.; Singh, A.; Schnur, J. M. Proc. Natl. Acad. Sci. U.S.A. 1996, 93, 1294312946. 
(37) Shimizu, T.; Hato, M. Biochim. Biophys. Acta 1993, 1147, 5058. 


\section{Supporting Information for}

Shedding Light on Helical Microtubules: Real-Time Observations of Microtubule SelfAssembly by Light Microscopy

Hee-Young Lee, Hyuntaek Oh, Jae-Ho Lee and Srinivasa R. Raghavan*

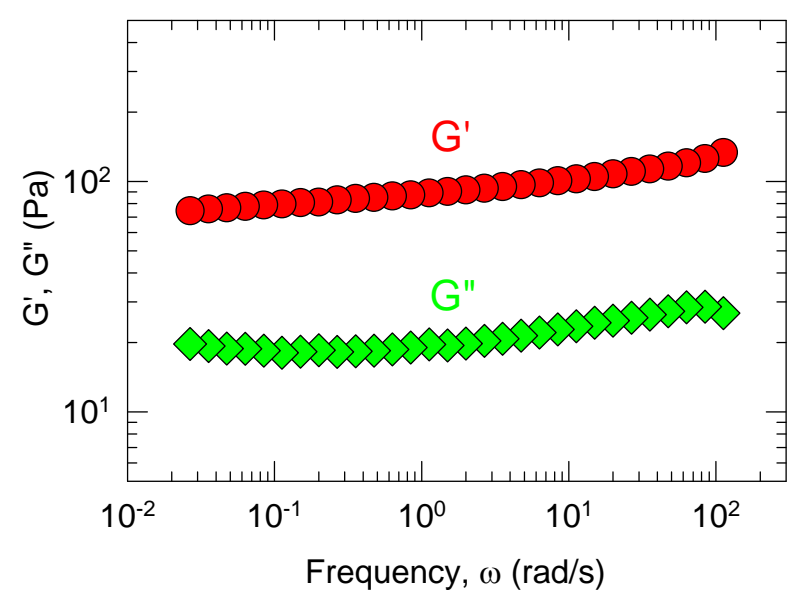

Figure S1. Rheology of a "vesicle gel" sample containing $11 \mathrm{wt} \%$ PCDA and $11 \mathrm{wt} \%$ geraniol. The data are from a dynamic frequency sweep and show the elastic modulus $G^{\prime}$ and the viscous modulus $G^{\prime \prime}$ as functions of frequency. We note that $G^{\prime}$ and $G^{\prime \prime}$ are nearly independent of frequency and that $G^{\prime}>G^{\prime \prime}$. Thus, the data reflect the gel-like (elastic) behavior of the sample. Similar homogeneous gels are obtained when $11 \mathrm{wt} \%$ PCDA is combined with 10-12 wt\% geraniol; at higher or lower geraniol concentrations, the samples separate into two phases rather than form a homogeneous gel.

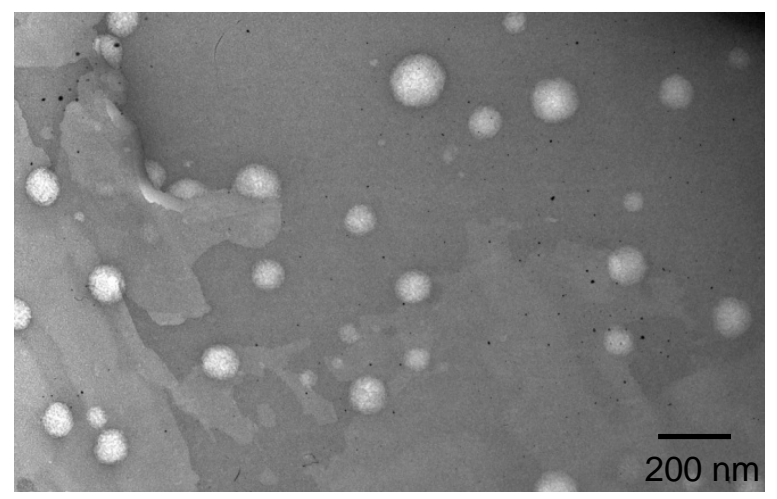

Figure S2. TEM image of vesicles in a sample obtained by $100 \mathrm{X}$ dilution of the PCDA/geraniol vesicle gel (30 min after dilution). The vesicles are negatively stained by uranyl acetate. 

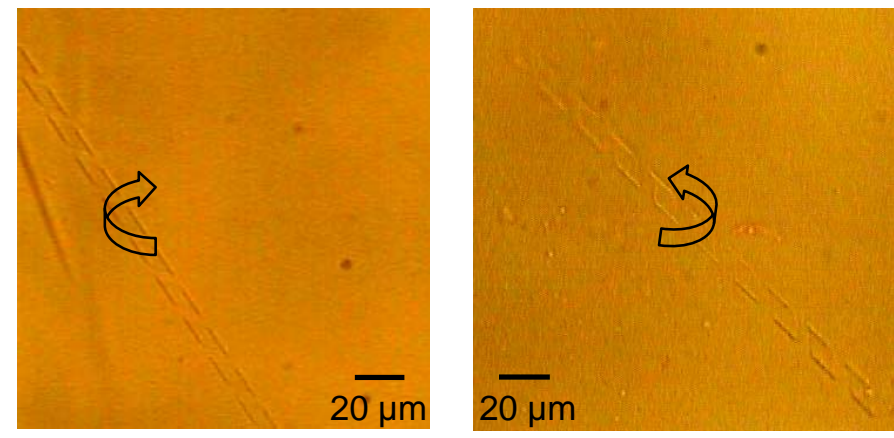

Figure S3. Optical micrographs of a left-handed helical ribbon (left) and a right-handed helical ribbon (right).
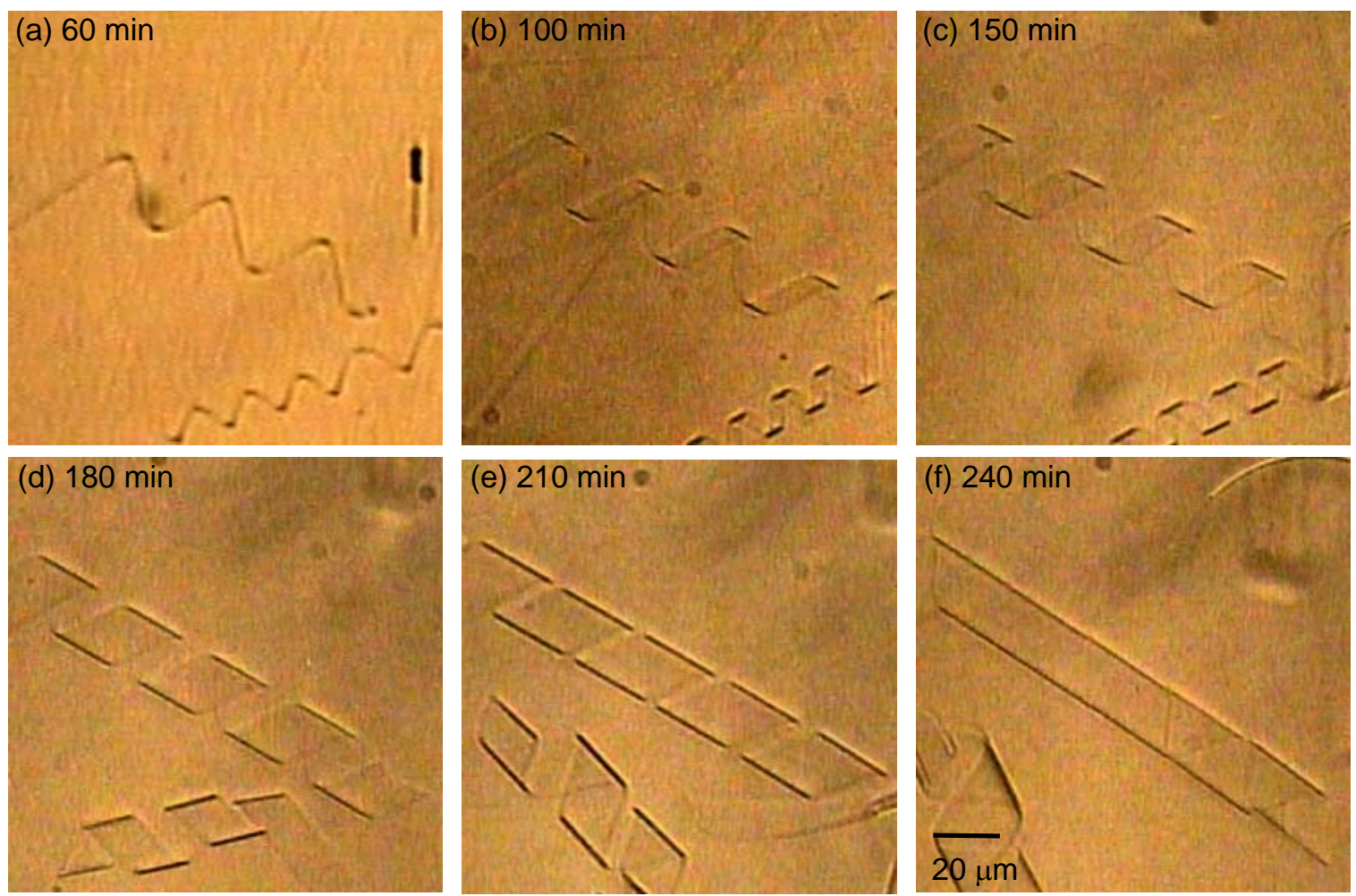

Figure S4. Optical micrographs in real time tracing the transformation of a helical ribbon to a closed hollow tubule. The images are similar to those in Figure 4 except that the ribbon is of much larger diameter in the present case. (a) 60 min after dilution of the PCDA/geraniol gel, a thin helical ribbon (29.6 $\mu \mathrm{m}$ pitch and a $17.5 \mu \mathrm{m}$ diameter, corresponding to a pitch angle of $28.3^{\circ}$ ) is identified. This helical ribbon grows longer and wider, as shown by the images at $100 \mathrm{~min}$ (b), $150 \mathrm{~min}$ (c), $180 \mathrm{~min}$ (d), and $210 \mathrm{~min}$ (e). As the width increases, the gaps between the turns are eliminated, resulting in a closed hollow tubule around $240 \mathrm{~min}$ (f). 

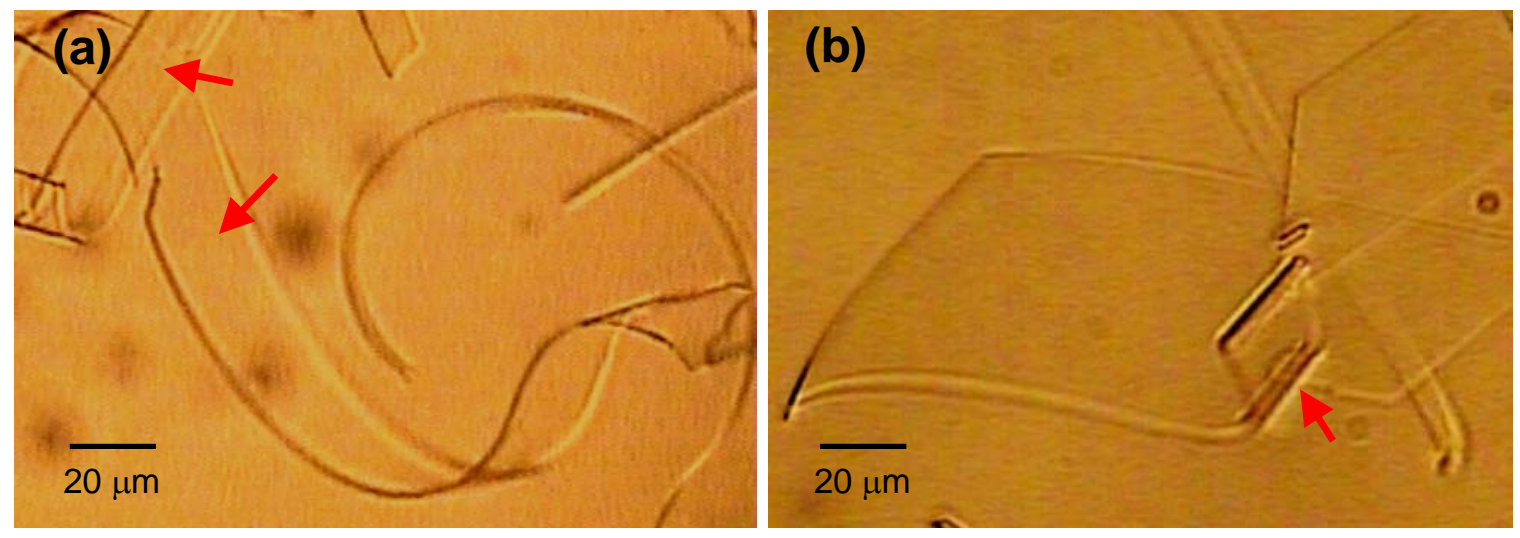

Figure S5. Optical microscope images showing some of the plate-like structures formed by unraveling of tubules. In (a) we see two large plate or sheet-like ribbon structures (arrows), much like the one seen in Figure 6e in the paper. Both structures appear to be rather stiff. In (b), the structure is wider and more flat, i.e., it looks more like a stiff plate. Note also that this structure is torn or broken at the point indicated by the arrow. We expect that the plates further break apart into discrete pieces as time progresses.
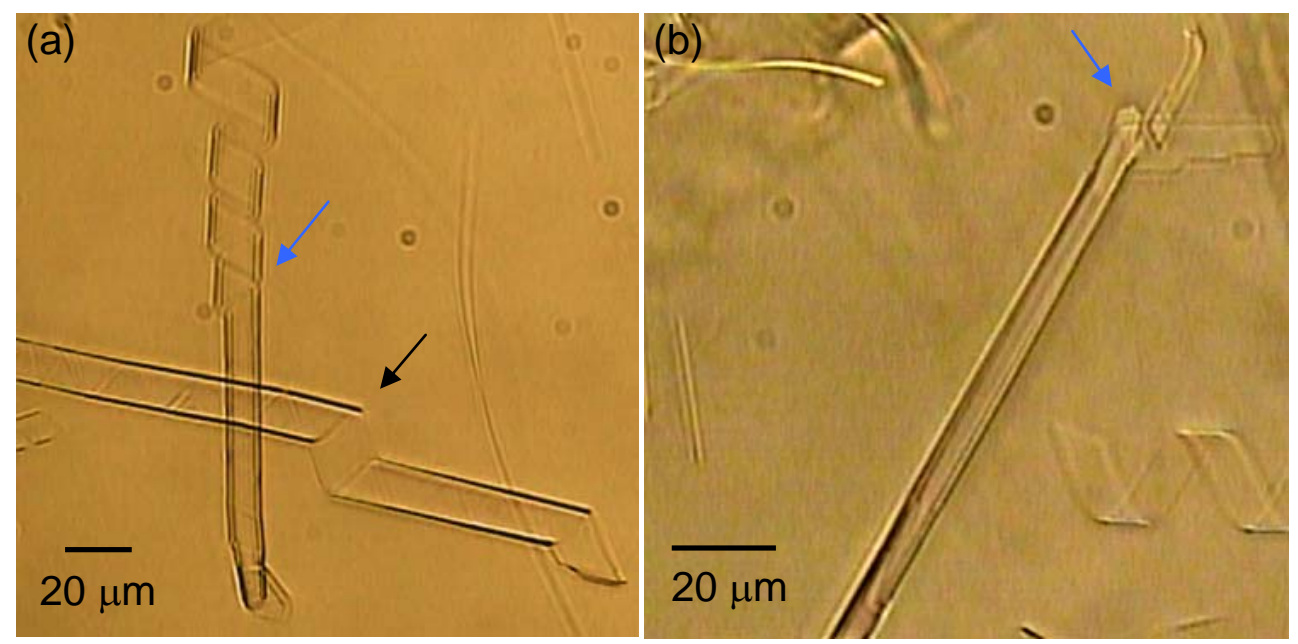

Figure S6. Optical micrographs showing different modes of unwinding or unraveling in the case of closed tubules. Most tubules unravel at a fracture or kink somewhere in the middle, as was shown in the main text in Figure 6. This is also seen in (a) for one tubule at the point marked by the black arrow. However, in the case of the second tubule in (a), the tubule unravels at one end, as shown by the blue arrow. In both these cases, the unraveling results in an unwound helical ribbon that widens and transforms into a stiff, brittle sheet or plate. The image in (b) shows another tubule that is also unraveling at one end (blue arrow); moreover, the unraveled portion is more like a sheet than a ribbon. 